This item was submitted to Loughborough's Research Repository by the author.

Items in Figshare are protected by copyright, with all rights reserved, unless otherwise indicated.

\title{
Rational design of photoelectrochemical cells towards bias-free water splitting: Thermodynamic and kinetic insights
}

PLEASE CITE THE PUBLISHED VERSION

https://doi.org/10.1016/j.jpowsour.2020.228113

PUBLISHER

Elsevier

VERSION

AM (Accepted Manuscript)

PUBLISHER STATEMENT

This paper was accepted for publication in the journal Journal of Power Sources and the definitive published version is available at https://doi.org/10.1016/j.jpowsour.2020.228113

LICENCE

CC BY-NC-ND 4.0

\section{REPOSITORY RECORD}

Zhang, Hao, H Wang, and Jin Xuan. 2020. "Rational Design of Photoelectrochemical Cells Towards Bias-free Water Splitting: Thermodynamic and Kinetic Insights”. Loughborough University. https://hdl.handle.net/2134/12238103.v1. 
Rational design of photoelectrochemical cells towards bias-free water splitting:

\section{Thermodynamic and kinetic insights}

Hao Zhang, ${ }^{1, *}$, Huizhi Wang ${ }^{2, *}$, Jin Xuan ${ }^{1, *}$

${ }^{1}$ Department of Chemical Engineering, Loughborough University, Loughborough, UK, LE11

$3 \mathrm{TU}$

${ }^{2}$ Department of Mechanical Engineering, Imperial College London, London, UK, SW7 2AZ

*Corresponding author: h.zhang2@1obro.ac.uk; huizhi.wang@imperial.ac.uk;

j.xuan@lboro.ac.uk 


\begin{abstract}
Photoelectrochemical (PEC) water splitting offers a promising way to produce hydrogen and harvest solar energy, however, its low efficiency has made it less economically attractive than other hydrogen production methods. Herein we present a numerical model of PEC cells considering quasi-fermi level splitting and interfacial kinetics to understand the charge transfer process and explore the approaches to increase the energy conversion efficiency. The non-linear change of photocurrent with light intensity under concentrated illumination is for the first time captured by a model. Based on the model, the operation regions of a PEC cell are mapped. Pathways to further promote the energy efficiency of PEC are proposed from the aspect of kinetics and thermodynamics. A new method that enables a precise evaluation of the theoretical boundaries of energy conversion efficiency of photocatalysts is developed taking into account the thermodynamics barrier.
\end{abstract}

Keywords: photoelectrochemical cell, concentrated sunlight, water splitting, numerical model, kinetic bias, thermodynamic bias. 


\section{Introduction}

Solar energy is regarded as the ultimate solution for sustainable energy supply for human society since it is the very original source of most energy forms on earth. As a significant utilization and storage approach of solar energy, the production of hydrogen through water splitting driven by sun lights has received considerable research attention. Photoelectrochemical (PEC) cells are regarded as a simple and highly integrated technology allowing a good product separation and direct light absorption at photoelectrode[1]. The theoretical maximum of solar-to-hydrogen conversion efficiency (STH) of PEC for water splitting is $47.3 \%$ (see SI for detail). It is suggested that the solar-assisted water splitting system can only achieve net primary energy balance if the STH exceeds the benchmark of $3-5 \%[2,3]$, and STH needs to reach the threshold of $10 \%$ to be commercially viable[4]. In regard to PECs, the index of applied bias photon-to-current efficiency (ABPE) is commonly used to assess the energy conversion efficiency instead of $\mathrm{STH}$, because a bias potential is normally required in PECs. In contrast, STH is an efficiency index under zero bias conditions, and thus widely applied in photovoltaic-electrolyzer (PV-E). Although the definitions and applications of ABPE and STH are different (see SI for detail), they both represent the ratio of net energy generation to the solar energy received. From this perspective, they are comparable. Over the past decade, substantial efforts have been devoted to developing efficient and robust photoelectrodes for PEC. Advanced visible-light responsive materials for photoelectrode has been proposed through crystalline doping, morphology manipulating, and particular treatment[5-9]. Heterojunction photoelectrodes combining n-type and p-type semiconductors have been developed to suppress the recombination of electron-hole pairs[10, 11]. Novel co-catalysts have 
been applied to accelerate the kinetics of water oxidation reaction on the surface of semiconductor[12, 13]. Unfortunately, the ABPE of present PECs for water splitting is struggling under 3\%[14-19]. The low ABPE performance of PECs is attributed to the application of the bias, as indicated in Eq. S1. As a matter of fact, the light saturating current density of photoelectrode is usually obtained at a rather positive potential. Take $\mathrm{BiVO}_{4}$-based photoanode as an instance, although larger than $5 \mathrm{~mA} \mathrm{~cm} \mathrm{~cm}^{-2}$ of current performance was performed, a bias of more than $1 \mathrm{~V}$ was necessary to drive the charge transfer and achieve such current density[20]. The use of bias significantly decreases the ABPE.

Nevertheless, the existing PEC devices often rely on the bias potential to accelerate the watersplitting process. As depicted in Fig. 1, applying bias can contribute to the reaction rate in three ways: (i) part of bias contributes to establishing the space charge layer in semiconductor to overcome the recombination of electron-hole pairs either at the surface or in the bulk of semiconductor and accelerate the charge transfer ( $\eta_{\text {bending }}$ ). (ii) Bias provides the overpotential to promote the surficial electrochemical kinetics at the counter electrode $\left(\eta_{c}\right)$. (iii) A portion of bias is inevitable to compensate for the thermodynamic barrier between the flat band potential of semiconductor and the equilibrium potential of the counter electrode, and initiate the charge transfer from photoanode to cathode $\left(\eta_{\text {onset }}\right)$. The $\eta_{\text {bending }}$ and $\eta_{c}$ are regarded as kinetic biases, and $\eta_{\text {onset }}$ is regarded as thermodynamics bias. Typically, since the current density of PEC is low at the current stage, i.e., around $10^{-1} \mathrm{~A} \mathrm{~m}^{-2}$, the ohmic loss within the electrolyte is in the magnitude of $10^{-4}$, which can be ignored, as shown in Fig. S1. Besides, due to the fast kinetics at counter electrode, the $\eta_{c}$ can also be neglected, as shown in Fig. S1. 
In order to meet the economic requirement of $\mathrm{PEC}$, two options are available. One is to decrease the benchmark by reducing the cost, the other is to further promote the ABPE of PECs. For the first option, concentrated solar irradiation is considered as a practical way of providing opportunities on further pressing down the cost of PEC and improvement in hydrogen yield [21, 22]. This strategy is valid only if PECs show linear photocurrent increasement with light intensity, which maintains the ABPE at an unchanged value. However, the favorable proportional photocurrent response only lies in low light concentration region (normally less than 3 suns)[23, 24], while a power function was observed in the highly concentrated region (up to more than 50 suns)[25], which, on the contrary, reduces the ABPE. The existing explanation attributes this undesirable photocurrent behavior to the higher recombination induced by increased intensity[25]. However, to date, no analytic understanding has yet been presented.

For the second option, sustained effort should be taken to continuously reduce either kinetic or thermodynamic bias applied in the system. Intensive researches have been carried out to develop advanced photoelectrode to reduce kinetic bias, while no quantitative strategy exists to guide efficient material development. In addition, the importance of thermodynamic bias has been overlooked. As a result, the traditional judgment on the theoretical energy conversion efficiency of photocatalyst based on STH calculation has been overestimated, which leads to a mislead on the selection and design of photocatalyst.

In this study, a multiphysics model of PEC which considers quasi-fermi level splitting and interfacial kinetics at photoelectrode was presented. To our best knowledge, this is the first model which gives a reliable photocurrent prediction under concentrated illumination. Based on the 
model, the operation regions are clarified through identifying the charge transfer determining step. Guidelines for material development aiming to reduce both kinetic bias and thermodynamic bias are proposed based on numerical analyses toward developing eco-attractive PEC. The theoretical efficiency of the photocatalyst was reevaluated by considering the factor of thermodynamic bias. This work provides a clear mechanistic understanding of photocurrent response on concentrated sunlight operation, and also gives guidelines on choosing and developing photoelectrode.

\section{Modeling methods}

\subsection{Physicochemical problem statement}

Fig. 1 shows a scheme of the flow PEC cell that was investigated in the study. The PEC cell consists of a $\mathrm{TiO}_{2}$ photoanode and a platinum cathode separated by a polymer electrolyte membrane was chosen for the model, because it is the most representative PEC system for water splitting. The flow configuration of the reactor gives the advantage of effective removal of gaseous production which could eliminate the effect of bubbles and simplify the flow characteristics of electrolyte. Therefore, it is reasonable to ignore the bubble effects in PEC flow cells. When illuminated by responsive light, electron-hole pairs are generated at photoanode, which causes the quasi-fermi level splitting. Driven by the potential difference in the space charge layer, electrons flow to the external circuit while holes transfer towards the electrode/electrolyte interface. Gaining energy from bias, the photo-induced electrons transfer further to cathode to complete the hydrogen evolution reaction (HER). The holes at the interface will participate in the oxygen evolution reaction (OER). In the model, the photocatalyst is regarded as homogeneous and the morphology 
of photocatalyst is not considered.

The water-splitting process follows the reaction equations.

Anode:

$$
\begin{gathered}
\mathrm{TiO}_{2} \stackrel{h v}{\longrightarrow} h^{+}+e^{-} \\
2 \mathrm{H}_{2} \mathrm{O}+h^{+} \rightarrow \mathrm{O}_{2}+4 H^{+}
\end{gathered}
$$

Cathode:

$$
2 H^{+}+2 e^{-} \rightarrow H_{2}
$$

\subsection{Photocurrent prediction}

PEC water splitting depends on two tandem physicochemical processes at photoanode, i.e., lightinduced carrier generation and transfer occurring within semiconductor and OER taking place at the interface of semiconductor/electrolyte.

The photocurrent induced by the transfer of photo-induced holes from inside of the electrode to the surface is expressed using a modified Gärtner-Butler equation to take into account the recombination effect.

$$
j_{p h}=\Phi_{\text {damping }} \Phi_{\text {re,surf }} e I\left[1-\frac{\exp \left(-\alpha \delta_{s c}\right)}{1+\alpha L}\right]
$$

where $I$ is the intensity of incident light $\left(\mathrm{m}^{-2} \mathrm{~s}^{-1}\right), \alpha$ is the absorption coefficient of photoanode $\left(\mathrm{cm}^{-}\right.$ $\left.{ }^{1}\right)$ and $L$ is the diffusion distance of hole $(\mathrm{m}) . \delta_{s c}$ is the thickness of the space charge layer $(\mathrm{m})$ which is given by[26].

$$
\delta_{s c}=\sqrt{\frac{2 \varepsilon \varepsilon_{0} \Delta \varphi_{s c}}{e N_{d}}}=\sqrt{\frac{2 \varepsilon \varepsilon_{0}\left(V-V_{f b}\right)}{e N_{d}}}
$$

where $\varepsilon$ is the dielectric constant, $\varepsilon_{0}$ is the permittivity of vacuum $\left(\mathrm{C} \mathrm{V}^{-1} \mathrm{~m}^{-1}\right), \Delta \varphi_{s c}$ is the difference 
of potential across the space charge layer $(\mathrm{V}), V$ is the potential of photoanode $(\mathrm{V}), V_{f b}$ is the flat band potential of semiconductor $(\mathrm{V}), e$ is the charge of electron $(\mathrm{C})$, and $N_{d}$ denotes the donor density of semiconductor $\left(\mathrm{m}^{-3}\right)$.

The diffusion distance of hole, $L$, is related to the charge lifetime $\tau$ (s) and diffusivity of holes $D$ $\left(\mathrm{m}^{2} \mathrm{~s}^{-1}\right)$ by

$$
L=\sqrt{D \tau}
$$

$L$ represents the bulk recombination property of a semiconductor.

In practice, concentration sunlight will lead to a remarkably increase of temperature, which thus results to the evaporation of electrolyte and the degeneration of photoelectrode. In order to eliminate this effect, a bandpass filter can be used to cut off the incident of infrared light. However, the bandpass filter could also cut off responsive light and reduce the intensity at the same time. In Eq. 4, a light damping factor $\Phi_{\text {damping }}$ of bandpass filter is added to take this effect into account. Since different bandpass filter has different cut off effect, $\Phi_{\text {damping }}$ is set one in this study, and can be adjusted according to the bandpass filter. Here, a PEC with ideal thermal management assumption was made and modeled.

A surface recombination coefficient $\Phi_{r e, s u r f}$ was used to consider the surface recombination effect, which is an exponential function of band bending $\Delta \varphi_{s c}$ in terms of two constants $\mathrm{A}$ and $\mathrm{B}$ described $[27,28]$

$$
\Phi_{r e, s u r f}=\frac{R_{t}}{R_{t}+R_{r}}=\frac{A \exp \left(B \cdot \Delta \varphi_{s c}\right)}{1+A \exp \left(B \Delta \varphi_{s c}\right)}
$$

where $R_{t}$ and $R_{r}$ denote the charge transfer resistance and recombination resistance at the surface 
of the semiconductor.

The OER current density was approximated by the Tafel equation

$$
j=j_{0, a} \exp \left(\frac{\beta^{a} F \eta_{a}}{R T}\right)
$$

where $j_{0, a}$ is the exchange current density $\left(\mathrm{A} \mathrm{m}^{-2}\right), \beta^{a}$ is the charge transfer coefficient of anodic reaction, $F$ is Faraday constant $\left(96485 \mathrm{C} \mathrm{mol}^{-1}\right), R$ is the gas constant $\left(8.314 \mathrm{~J} \mathrm{~mol}^{-1} \mathrm{~K}^{-1}\right)$, and $T$ is the thermodynamic temperature $(\mathrm{K})$. The interfacial overvoltage $\eta_{a}(\mathrm{~V})$ is defined as

$$
\eta_{a}=\varphi-E_{e q}^{O E R}
$$

where $E^{O E R}$ is the $\mathrm{pH}$-dependent equilibrium potential

$$
E_{e q}^{\text {OER }}=1.229-0.059 \mathrm{pH}
$$

In previous modeling studies[27, 28], the electrode potential of the electrode $\varphi$ is defined as $\varphi_{s}-\varphi_{l}$.

This is true for conventional metal electrodes. However, for an n-type semiconductor, $\varphi$ stands for the quasi-Fermi level of holes at the surface of the photoanode. In the dark, the Fermi level of semiconductor lies between the conduction band (CB) and valence band (VB) equilibrates with the Fermi level of electrolyte. Under irradiation, the Fermi level splits into two quasi-Fermi levels, $E_{f, n}$ and $E_{f, p}$, for interfacial electrons and holes, which are used to describe the nonequilibrium condition of semiconductor (Fig. 1). The OER becomes thermodynamically favorable only if the following energy condition is satisfied, in which the quasi-fermi level of holes is more positive than the equilibrium potential of OER.

$$
E_{f, p}>E_{e q}^{O E R}
$$

The overpotential of photoanode is 


$$
\eta_{a}=E_{f, p}-E_{e q}^{O E R}
$$

The quasi-Fermi levels of electrons and holes can be determined as follows.

$$
\begin{aligned}
& E_{f, n}=E_{f, s c}-\frac{k T}{e} \ln \left(\frac{n+\Delta n}{n}\right) \\
& E_{f, p}=E_{f, s c}+\frac{k T}{e} \ln \left(\frac{p+\Delta p}{p}\right)
\end{aligned}
$$

where $E_{f, s c}$ is the Fermi-level of semiconductor, $n$ and $p$ are the concentration of electrons and holes under dark, $\Delta n$ and $\Delta p$ are the concentration of the light-induced electrons and holes.

Therefore, $E_{f, p}$ can be obtained through substituting Eq. 13 into Eq. 14.

$$
E_{f, p}=E_{f, n}+\frac{k T}{e} \ln \left(\frac{n+\Delta n}{n} \cdot \frac{p+\Delta p}{p}\right)
$$

The concentrations of electron and hole in dark, i.e., $n$ and $p$, follow the following expression.

$$
n \times p=\left(N_{C} N_{V}\right) \exp \left(\frac{-E_{g}}{k T}\right)
$$

where $N_{C}$ is the effective state density at the lower edge of the conduction band $\left(\mathrm{m}^{-3}\right), N_{V}$ is the effective state density at the upper edge of the valence band $\left(\mathrm{m}^{-3}\right), E_{g}$ is the bandgap of semiconductor $(\mathrm{eV})$, which is $3.2 \mathrm{eV}$ for $\mathrm{TiO}_{2}$. In an n-type semiconductor, $n$ can be approximated as donor density $N_{d}$.

$N_{C}$ and $N_{V}$ are given by

$$
\begin{aligned}
& N_{C}=2\left[\frac{2 \pi m_{e f f}^{e} k T}{h^{2}}\right]^{\frac{3}{2}} \\
& N_{V}=2\left[\frac{2 \pi m_{\text {eff }}^{h} k T}{h^{2}}\right]^{\frac{3}{2}}
\end{aligned}
$$


where $m^{e}$ eff and $m^{h}$ eff are the effective mass of electron and hole, respectively.

The $E_{f, n}$ is given by.

$$
E_{f . n}=E_{c}+\frac{k T}{e} \ln \left(\frac{N_{C}}{n+\Delta n}\right)
$$

Normally, the donor density $n$ for $\mathrm{TiO}_{2}$ ranges from $10^{22} \mathrm{~m}^{-3}$ to $10^{26} \mathrm{~m}^{-3}$ [29]. Here, a magnitude of $10^{24} \mathrm{~m}^{-3}$ is used[9]. While $N_{C}$ can be calculated in the magnitude of $10^{26} \mathrm{~m}^{-3}$ by Eq. 17 , as shown in Table S2. $\Delta n$ can be estimated as the number of responsive photons from AM 1.5G, which is in the in the magnitude of $10^{20} \mathrm{~m}^{-3}$. Therefore, it is reasonable to approximate the quasi-Fermi level of electrons equals the penitential of the conduction band.

$$
E_{f . n}=E_{c}
$$

For $\mathrm{TiO}_{2}, n>>\Delta n, p<<\Delta p$, so Eq. 15 becomes.

$$
E_{f, p}=E_{f, n}+\frac{k T}{e} \ln \left(\frac{\Delta p}{p}\right)=E_{c}+\frac{k T}{e} \ln \left(\frac{\Delta p}{p}\right)
$$

At the interface of photoanode and electrolyte, the position of the conduction band is the flat band, this gives

$$
E_{f, p}=E_{f b}+\frac{k T}{e} \ln \left(\frac{\Delta p}{p}\right)
$$

Substituting Eq. 22 into Eq. 12, we have

$$
\eta_{a}=E_{f b}+\frac{k T}{e} \ln \left(\frac{\Delta p}{p}\right)-E_{e q}^{O E R}
$$

AM1.5G spectra have been used as the incident light. The intensity of incident light, $I$, is derived from the spectra of AM $1.5 \mathrm{G}$ through the following expression. 


$$
I=\Phi_{\text {optical }} \int \frac{I r}{h c_{\text {light }} / \lambda} d \lambda
$$

The overall optical losses $\Phi_{\text {optical }}$ are estimated at $25 \%[26]$.

Light decay within the $\mathrm{TiO}_{2}$ nanorods caused by light absorption was considered in this model. The distribution of light intensity is given by Beer-Lambert law.

$$
\frac{I(x)}{I}=\exp (-\alpha x)
$$

in which, $x$ is the transmission length in the direction of light travels (m). The absorption coefficient of $\mathrm{TiO}_{2}$ is derived from Ref. 26 according to the following expression.

$$
\alpha=\frac{a}{d \log e_{0}}
$$

where $a$ is the absorbance of $\mathrm{TiO}_{2}, d$ is the thickness of $\mathrm{TiO}_{2}$ layer $(\mathrm{cm}), e_{0}$ is the base of the natural logarithm.

In fact, the photocurrent should be determined by the absorbed fraction of light, $I-I(x)$, rather than the incident light intensity $I$. Hence, the Eq. 4 has been further modified as

$$
j_{p h}=\Phi_{r e, s u r f} e(I-I(x))\left[1-\frac{\exp \left(-\alpha \delta_{s c}\right)}{1+\alpha L}\right]
$$

The dielectric constant $\varepsilon$ of $\mathrm{TiO}_{2}$ is derived from the Mott-Schottky plot in Ref. 26.

$$
\frac{1}{C^{2}}=\left(\frac{2}{e \varepsilon \varepsilon_{0} N_{d}}\right)\left[\left(V-V_{f b}\right)-\frac{k T}{e}\right]
$$

in which, $C$ is the capacitance. Instead of using a fixed dielectric constant, the profile of $\varepsilon$ against the potential of the photoanode was applied in the model. 


\subsection{Cathode kinetics}

The cathode kinetics can be described by the use of Butler-Volmer equation

$$
j=j_{0, c}\left(\exp \left(\frac{\beta_{c} F \eta_{c}}{R T}\right)-\exp \left(\frac{\left(1-\beta_{c}\right) F \eta_{c}}{R T}\right)\right)
$$

where $j_{0, c}$ is the exchange current density of hydrogen evolution reaction (HER) at the interface of cathode/electrolyte $\left(\mathrm{A} \mathrm{m}^{-2}\right), \beta_{c}$ is the transfer coefficient of cathodic reaction. The overpotential is defined as

$$
\eta_{c}=\varphi-E_{e q}^{H E R}
$$

where $E^{H E R}$ eq is the $\mathrm{pH}$-dependent equilibrium potential

$$
E_{e q}^{H E R}=0-0.059 \mathrm{pH}
$$

Since the cathode is a conventional metal electrode, the electrode potential $\varphi$ is defined as $\varphi_{S}-\varphi_{l}$.

The charge transfer in the electrolyte and at the cathode is given by the ohmic law

$$
j=-\sigma \nabla \varphi
$$

where $\sigma$ is conductivity $(\mathrm{S} / \mathrm{m})$ of electrolyte and cathode.

\subsection{Species and charge transport in the electrolyte}

The electrolyte flow in the microchannel was modeled by solving the continuity equation and momentum conservation,

$$
\begin{gathered}
\nabla \cdot(\rho u)=0 \\
\nabla \cdot(\rho u u)=-\nabla \cdot \bar{p}+\nabla \cdot \tau
\end{gathered}
$$

where $\rho$ is the density of the fluid $\left(\mathrm{kg} \mathrm{m}^{-3}\right), u$ denotes the velocity vector, $\bar{p}$ is the pressure $(\mathrm{Pa})$, 
$\tau$ is the viscous stress tensor. The dissolved species $\left(\mathrm{O}_{2}\right.$ at the anode and $\mathrm{H}_{2}$ at the cathode $)$ in the electrolyte are calculated by solving the conservation of species

$$
\nabla \cdot\left(-\bar{D}_{i} \nabla c_{i}\right)+u \cdot \nabla c_{i}=R_{i}^{\prime}
$$

where $c$ refers to the concentration of each species $\left(\mathrm{mol} \mathrm{m}^{-3}\right), \bar{D}$ denotes diffusivity of species $\left(\mathrm{m}^{2} \mathrm{~s}^{-1}\right), R^{\prime}$ is the generation or consumption rate of species $\left(\mathrm{mol} \mathrm{m}^{-3} \mathrm{~s}^{-1}\right)$, which is related to current through Faraday's law. The governing equations and their associated boundary conditions are summarized in Table S1.

\subsection{Model parameters and numerical procedures}

The baseline values used for the numerical validation are summarized in Table S2. The numerical model was solved by COMSOL Multiphysics. A fully coupled direct solution procedure with Multifrontal Massively Parallel Solver (MUMPS) was applied to implement the calculation. The parametric sweep was used to calculate the current-potential performance curve.

\section{Results}

\subsection{Model validation}

Fig. 2a shows the photocurrent response with illumination intensity. Our model suggests a power correlation between photocurrent and sunlight intensity instead of linear relationship at high light intensity region, which agrees with the experiments findings from Bell et al.[25] and Carey et al.[30]. However, the conventional model based on Gärtner-Butler equation would result in a continuous linear current response with concentrated sunlight. This is because the interfacial 
kinetics at photoanode (OER) restricts the overall charge transfer rate responsive linearly, which is defined and calculated by Eq. 8 -Eq. 23. The simulated results at incident intensities of 1 sun and 2.3 suns are in good agreement with the experimental data obtained in the work of Wolcott et al.[26], as shown in Fig. 2b. It is notable that in the low intensity region $(<2 \sim 3$ suns), the power function correlations are close to the linear prediction derived from Gärtner-Butler equation, which indicates the validation of Gärtner-Butler equation in the low intensity region. However, in the highly concentrated illumination region, large deviation from the actual performance will be obtained by Gärtner-Butler equation. The results show a significant improvement in predictability has been achieved in this model. Further model validation can also be found in section 3.5 on modeling the photoelectrode behaviors with normal $\mathrm{TiO}_{2}$ and hydrogen treated $\mathrm{TiO}_{2}$, from which it can be found that the model is capable of accurately reflecting the effects of material properties (such as absorption and donor density) on the overall PEC performance.

\subsection{Understanding factors limiting ABPE in a PEC cell}

An effective way to increase photocurrent and reduce the cost of PEC panels is to use concentrated sunlight. Fig. 3a-3c shows how the photocurrent response with light power at circumstances with different interfacial OER kinetics. At the condition of sluggish interfacial OER kinetics, i.e., anodic exchange current density of less than $4 \times 10^{-11} \mathrm{~A} \mathrm{~m}^{-2}$ for this model, it is found that current performance sticks to the power function of light intensity, as shown in Fig. 3a. The correlation equation between light intensity $I$ and current density $j$ is in the following form with an $R^{2}$ value of 0.999 


$$
j=0.18 \cdot I^{0.5}
$$

When the charge transfer rate of OER at the surface of the semiconductor is lower than the maximum carrier transfer rate within the semiconductor, the overall current performance is limited by interfacial kinetics. As indicated in Eq. 23, the overpotential at photoanode rises with the number of light-induced holes, which leads to an improvement in OER as suggested in Eq. 8. Though the photocurrent increases with light intensity, ABPE drops sharply, as shown in Fig. 3a. This operation region is named kinetic-controlled region.

Integrating co-electrocatalyst with photoanode has been widely used to reduce the activation energy and enhance the kinetics of $\operatorname{OER}[12,31]$. The photocurrent of $\mathrm{TiO}_{2}$ was reported rising more than $120 \%$ by coating NiFe-based co-catalyst[32]. Furthermore, a disordered surface layer acting as co-catalyst can be formed at the surface of $\mathrm{TiO}_{2}$ after a treatment of electrochemical reduction, which obtained 2.2 times photocurrent[33]. With improved OER kinetics, the charge transfer rate of OER exceeds the maximum photocurrent of semiconductor, at which circumstance the overall current performance is determined by carrier transfer rate in the photoanode. Therefore, the photocurrent keeps climbing up linearly with light intensity until the OER kinetics becomes rate-limiting step again. This linear operation region is light-controlled region. In this model, at the circumstance with moderate OER kinetics, i.e., the anodic exchange current density between $4 \times 10^{-11} \mathrm{~A} \mathrm{~m}^{-2}$ to $2.75 \times 10^{-10} \mathrm{~A} \mathrm{~m}^{-2}$, the current performance relation with light power can be divided into two regions, a linearly increasing part and a power increasing part, as shown in Fig. 3b. As a result, the ABPE keeps a constant at the linear region, then declines in the power region. Take anodic exchange current density of $1.5 \times 10^{-10} \mathrm{~A} \mathrm{~m}^{-2}$ as an example, current performance improves 
parallelly with light power until 15 suns. Further improvement in light intensity results in a power correlation.

$$
\begin{gathered}
j=0.17 \cdot I \quad\left(I \leq 15 \times \mathrm{AM} 1.5, R^{2}=1\right) \\
j=0.68 \cdot I^{0.5} \quad\left(I>15 \times \mathrm{AM} 1.5, R^{2}=1\right)
\end{gathered}
$$

In the circumstance with rapid OER kinetics (anodic exchange current density of $3 \times 10^{-10} \mathrm{~A} \mathrm{~m}^{-2}$ ), as shown in Fig. 3c, the overall current at the electrode is determined by the photocurrent at semiconductor and is linear with light intensity. Therefore, an unchanged ABPE is obtained at the whole range of light irradiation. Fig. 3d shows the working regions of PEC categorized by the interfacial kinetics and light intensity.

\subsection{Bias reduction through designing electrode thickness}

The first attempt to increase the ABPE of PEC is promoting the current performance of photoanode, and thus reduce the bias required for electron-hole separation in the semiconductor. As absorbed by the semiconductor, light intensity decays exponentially in the direction of propagation, as shown in Fig. 4a.

In order to harness as much light as possible, thicker photocatalyst should be utilized. Fig. 3b shows the effect of the semiconductor thickness on photocurrent performance. The photocurrent climbs with the thickness of $\mathrm{TiO}_{2}$ growing and gradually reaches a plateau. More than $90 \%$ improvement in current performance could be achieved by thickening $\mathrm{TiO}_{2}$ layer from $0.5 \mu \mathrm{m}$ to $4 \mu \mathrm{m}$. As a result, the ABPE follows the same trend with photocurrent, as shown in Fig. 4b. Fig. $4 \mathrm{c}$ shows the light absorption fraction within the semiconductor, which indicates that the 
responsive light between $280 \mathrm{~nm}$ to $400 \mathrm{~nm}$ has almost been absorbed at the thickness of $4 \mu \mathrm{m}$. The same dependence of photocurrent on length of $\mathrm{TiO}_{2}$ nanorods was reported in the work of Hwang et al.[34]

\subsection{Bias reduction through designing material properties}

The limiting photocurrent caused by photon flux can be calculated from the spectra of AM1.5G based on the following equation

$$
j_{\text {photo,limit }}=e \Phi_{\text {optical }} \Phi_{\text {damping }} \int_{\lambda} \frac{I r}{h c / \lambda} d \lambda
$$

in which, $I r$ is the spectral irradiance of AM1.5G $\left(\mathrm{W} \mathrm{m}^{-2} \mathrm{~nm}^{-1}\right), e$ is the elementary charge (1.602 $\left.\times 10^{-19} \mathrm{~J}\right), h$ is the Planck's constant $\left(6.626 \times 10^{-34} \mathrm{~J} \mathrm{~s}\right)$, clight is the speed of light $\left(2.998 \times 10^{8} \mathrm{~m} \mathrm{~s}^{-1}\right)$, $\lambda$ is the wavelength (m), $\Phi_{\text {optical }}$ denotes the optical losses caused by light reflection of conductive glass and $\mathrm{TiO}_{2}$, absorption and scattering of conductive glass.

The calculated value of the theoretical photocurrent limit is $10.1 \mathrm{~A} \mathrm{~m}^{-2}$ regarding the responsive wavelength range $280 \sim 400 \mathrm{~nm}$ after considering optical loss caused by FTO glass. Notably, the photocurrent of less than $0.2 \mathrm{~A} \mathrm{~m}^{-2}$ can be reached at a thickness of $4 \mu \mathrm{m}$, as shown in Fig. $4 \mathrm{c}$, which indicates that photoanode is far away from the photon limiting region.

It is instinctive to regard the absorption performance of semiconductor as a main factor attributes to the huge gap between the photon limiting current and the obtained value. Therefore, the effect of improving absorption performance on current performance has been investigated. As shown in

Fig. 5a and 5b, higher absorption factors indeed results in a higher current until all the responsive 
light was absorbed. However, the intensification of performance is limited. It is found that both methods of thickening catalyst and improving absorption coefficient leads to the same outcome, which is capturing as much responsive light as possible, as indicated in Fig. 5c. Therefore, light absorption performance is not the main current determinant factor of a certain semiconductor.

Hole diffusion length is another critical factor affecting current performance. Fig. 5a gives the current curves at various hole diffusion lengths. In reality, the diffusion performance of charge carriers can be promoted through suppressing the recombination of electron-hole pairs. With prolonging the diffusion length of holes, which means suppressing the recombination of electronhole, it shows dramatical improvement in current performance. In the circumstance of $10 \mu \mathrm{m}$ in diffusion length, the current density of $9.4 \mathrm{~A} \mathrm{~m}^{-2}$ can be obtained, which approaches the light limiting current. The maximum $\mathrm{ABPE}$ of $\mathrm{TiO}_{2}$ can be achieved at around $0.3 \%$, as shown in Fig. $5 b$.

To further confirm the charge transfer property of semiconductor is the key factor that prevents photocurrent from reaching its theoretical photocurrent limit, the effect of donor density $N_{d}$ on photocurrent was investigated. Donor density $N_{d}$ plays an essential role in the photocurrent performance of photoelectrode. The higher $N_{d}$ enables more effective spatial charge separation, which results in the suppression of electron-hole recombination[35]. In practice, the donor density of photocatalyst relies on the preparation method and postprocessing, and can be improved by vapor deposition[36], doping[37], hydrogen-treatment[9], electrochemical treatment[16] and ultraviolet curing[38].

To further verify the above analysis, hydrogen-treated $\mathrm{TiO}_{2}\left(\mathrm{H}-\mathrm{TiO}_{2}\right)$ photoanodes were chosen as 
the case studied. It is selected because a large number of oxygen vacancies are generated in the semiconductor during the hydrogen treatment serving as donors without introducing any dopants into $\mathrm{TiO}_{2}$ structure[9]. The model has been validated with experimental data from the work of Wang et.al.[9], as shown in Fig. S2. Table 1 shows the experimental values of the absorption coefficient and donor density, and values of hole diffusion lengths derived from the model. Comparing with $\mathrm{TiO}_{2}$, it is found that recombination has been efficiently suppressed through increasing donor density in $\mathrm{H}-\mathrm{TiO}_{2}$. Besides, light absorption property has been enhanced with donor density as well. In spite of the continuous improvement in light absorption performance, the hole diffusion length drops with donor density further increasing from $2.1 \times 10^{28}$ to $7.8 \times 10^{28} \mathrm{~m}^{-3}$, which attributes to the degeneration of photocurrent. It is because that in high donor density situation, the vacancies serve as trapping sites and become recombination centers for electron-hole pairs[39]. The effect of donor density on the lifetime of photogenerated holes has been experimentally confirmed by Elbanna et al. [40].

Table 1 Optical and charge transfer properties of $\mathrm{TiO}_{2}$ and $\mathrm{H}-\mathrm{TiO}_{2}$

\begin{tabular}{|c|c|c|c|c|c|}
\hline Parameters & $\mathrm{TiO}_{2}$ & $\mathrm{H}-\mathrm{TiO}_{2} 350^{\circ} \mathrm{C}$ & $\mathrm{H}-\mathrm{TiO}_{2} 400^{\circ} \mathrm{C}$ & $\mathrm{H}-\mathrm{TiO}_{2} 450^{\circ} \mathrm{C}$ & Source \\
\hline Normalized $\alpha$ & 1 & 1.38 & 1.47 & 2.29 & Wang et al.[9] \\
\hline$N_{d}\left(\mathrm{~m}^{-3}\right)$ & $5.3 \times 10^{24}$ & $2.1 \times 10^{28}$ & $3.24 \times 10^{28}$ & $7.8 \times 10^{28}$ & Wang et al.[9] \\
\hline$L(\mathrm{~m})$ & $2 \times 10^{-7}$ & $8.8 \times 10^{-7}$ & $6.5 \times 10^{-7}$ & $2.8 \times 10^{-7}$ & Fitted \\
\hline
\end{tabular}

Based on the above analyses, it can be concluded that the transport property of the charge carrier is determined as the primary limiting factor on photocurrent. The result indicates that the future 
development of advanced photoanode should pay more attention to electron-hole recombination suppression.

\subsection{Bias reduction through tuning the onset bias}

Fig. 6 maps the ABPEs of PECs based on three photoanodes, i.e., $\mathrm{TiO}_{2}(3.2 \mathrm{eV}), \mathrm{WO}_{3}(2.7 \mathrm{eV})$ and $\mathrm{BiVO}_{4}(2.4 \mathrm{eV})$. The achieved efficiencies of currently developed photoelectrodes were located on the map $[9,26,41,42]$. As expected, applying photocatalyst with narrow bandgap pushes up the theoretical ceiling of ABPE. The figure indicates that the theoretical maximum ABPE can only be achieved at onset bias with rapid electron-hole separation, which indicates that the onset bias represents the thermodynamically minimum bias required in the system.

It is found that a negative shift of flat band is favorable for reducing the onset bias. The position of flat band is determined by multiple factors. On one hand, it directly relies on the material of photocatalyst. On the other hand, even for a certain kind of photocatalyst, the flat band will be affected by crystal structure[43, 44], preparation method[38] and material modification[45]. Therefore, tuning of the flat band should be an important research aspect for promoting the efficiency of PEC.

\subsection{Theoretical energy conversion efficiency evaluation}

Fig. 7 maps the thermodynamically maximum energy efficiency of PEC with the effect of onset bias. The theoretical upper boundary of $\mathrm{CB}$ is given when VB equals to the equilibrium potential of OER (band position i), in which occasion, the semiconductor can carry out OER. The theoretical 
bottom boundary of the conduction band of semiconductor is given as the equilibrium potential of OER (band position ii). When the conduction band is more positive than OER, the theoretical onset bias will be larger than $1.23 \mathrm{~V}$, which leads to a failure of water splitting. When CB locates above HER potential, the $\mathrm{CB}$ is more negative than HER potential which will result in automatic electron flow from photoanode to cathode and no onset bias is needed (band position i). Under this situation, $\mathrm{ABPE}$ and STH become equivalent. While, when CB positions beneath HER potential, onset bias is thermodynamically imperative to drive the charge transfer to cathode (band position iii), which leads to a reduction on ABPE.

In the previous understanding[46], bandgap was considered as the sole factor affects theoretical STH of PEC, which is true only when CB is more negative than HER potential. The influence of band position has been overlooked. Taking $\mathrm{BiVO}_{4}$ with a band gap of $2.4 \mathrm{eV}$ as instance, according to Eq. S2, the theoretical STH without considering the onset bias effect is 9.3\%. However, the CB of $\mathrm{BiVO}_{4}$ is $0.46 \mathrm{~V}$ vs $\mathrm{NHE}$ at $\mathrm{pH}=0$ [47], i.e., $0.46 \mathrm{~V}$ more positive than the equilibrium potential of HER (similar to case iii in Fig 7), representing a thermodynamic barrier hindering electrons transfer to cathode, which must be compensated by onset bias. After taking this effect into account, the theoretical ABPE reduces to 5.8\%. It indicates that previous calculation overestimates the maximum efficiency of $\mathrm{BiVO}_{4}$ by $60 \%$. Similar results also apply to other semiconducting materials, such as $\alpha-\mathrm{Fe}_{2} \mathrm{O}_{3}$ with band gap of $2.1 \mathrm{eV}$ (12.6 \% of ABPE rather than $15.3 \%$ of $\mathrm{STH})[48], \mathrm{WO}_{3}$ with band gap of $2.7 \mathrm{eV}(3.3 \%$ of $\mathrm{ABPE}$ rather than $4.9 \%$ of $\mathrm{STH})$ [49]. Therefore, it is crucial to consider both the band position and band gap in choosing and developing photocatalyst in the future. 


\section{Discussion}

This work presents a novel predictive framework on PEC water splitting considering quasi-fermi level splitting in semiconductor and interfacial kinetics at photoelectrode. The model for the first time provides a reliable prediction on photocurrent response at highly concentrated sunlight operation. It also revealed the important underlying physical principles behind the the power function behavior of photocurrent at high light intensity, which can be attributes to the interfacial charge transfer process. Rational design guidelines are derived for PEC under concentrated irradiation. To maintain an unreduced ABPE, the PEC should be designed to operate in lightcontrolled region. Otherwise, a trade-off between ABPE and sunlight intensity will occur.

With the aim to achieve bias-free PEC water splitting, guidelines on developing photoelectrode materials are proposed based on the model to reduce the kinetic and thermodynamic biases. Promoting the charge transfer performance is found critical to reduce kinetic bias, which can be achieved by manipulating material properties like donor density. Take $\mathrm{TiO}_{2}$ photoelectrode for instance, nearly 5 times increasement in ABPE can be achieved through tuning donor density. On the other hand, optimizing light absorption performance and geometry dimension of photocatalyst are found helpful to promote photocurrent performance and thus promote energy efficiency. However, the potential of improvement with this method is limited. In addition, negative shift of conduction band through tuning the band position of photocatalyst helps to reduce the thermodynamic bias. The study highlighted the importance of developing onset-bias free photoelectrode material in efficiency improvement of PEC water splitting. The work also corrects 
the previous understanding which overestimated the theoretical energy conversion capacity of a semiconductor material used in PEC water splitting, by considering the effect of thermodynamic barrier, i.e., onset bias. This paper advances fundamental understandings on charge transfer processes within PEC system, and unveils the underlying processes hindering the enhancement of PEC efficiency. It therefore will inspire the wider solar fuel research community on future material and device developments towards the ultimate target of commercially-viable sustainable hydrogen production.

\section{Supplementary information}

Supplementary information is available for this paper.

\section{Acknowledgements}

The research reported in this paper is supported by UK Engineering and Physical Sciences Research Council (EPSRC) via grant number EP/R012164/2 and EP/S000933/1.

\section{References:}

[1] C. Jiang, S.J.A. Moniz, A. Wang, T. Zhang, J. Tang, Chemical Society Reviews, 46 (2017) $4645-4660$.

[2] P. Zhai, S. Haussener, J. Ager, R. Sathre, K. Walczak, J. Greenblatt, T. McKone, Energy \& Environmental Science, 6 (2013) 2380-2389.

[3] R. Sathre, J.B. Greenblatt, K. Walczak, I.D. Sharp, J.C. Stevens, J.W. Ager, F.A. Houle, Energy 
\& Environmental Science, 9 (2016) 803-819.

[4] B.A. Pinaud, J.D. Benck, L.C. Seitz, A.J. Forman, Z. Chen, T.G. Deutsch, B.D. James, K.N.

Baum, G.N. Baum, S. Ardo, H. Wang, E. Miller, T.F. Jaramillo, Energy \& Environmental Science, 6 (2013) 1983-2002.

[5] S. Wang, G. Liu, L. Wang, Chemical reviews, (2019).

[6] J. Joy, J. Mathew, S.C. George, International Journal of Hydrogen Energy, 43 (2018) 48044817.

[7] A.G. Tamirat, J. Rick, A.A. Dubale, W.-N. Su, B.-J. Hwang, Nanoscale Horizons, 1 (2016) 243-267.

[8] T. Hisatomi, J. Kubota, K. Domen, Chemical Society Reviews, 43 (2014) 7520-7535.

[9] G. Wang, H. Wang, Y. Ling, Y. Tang, X. Yang, R.C. Fitzmorris, C. Wang, J.Z. Zhang, Y. Li, Nano Letters, 11 (2011) 3026-3033.

[10] S.J.A. Moniz, S.A. Shevlin, D.J. Martin, Z.-X. Guo, J. Tang, Energy \& Environmental Science, $8(2015) 731-759$

[11] K. Zhang, M. Ma, P. Li, D.H. Wang, J.H. Park, Advanced Energy Materials, 6 (2016) 1600602.

[12] C. Ding, J. Shi, Z. Wang, C. Li, ACS Catalysis, 7 (2017) 675-688.

[13] I. Roger, M.A. Shipman, M.D. Symes, Nature Reviews Chemistry, 1 (2017) 0003.

[14] R. Zhang, F. Ning, S. Xu, L. Zhou, M. Shao, M. Wei, Electrochimica Acta, 274 (2018) 217 223.

[15] F. Ning, M. Shao, S. Xu, Y. Fu, R. Zhang, M. Wei, D.G. Evans, X. Duan, Energy \& Environmental Science, 9 (2016) 2633-2643. 
[16] S. Wang, P. Chen, J.-H. Yun, Y. Hu, L. Wang, Angewandte Chemie International Edition, 56 (2017) 8500-8504.

[17] G. Liu, S. Ye, P. Yan, F. Xiong, P. Fu, Z. Wang, Z. Chen, J. Shi, C. Li, Energy \& Environmental Science, 9 (2016) 1327-1334.

[18] T.W. Kim, Y. Ping, G.A. Galli, K.-S. Choi, Nature Communications, 6 (2015) 8769.

[19] S. Wang, P. Chen, Y. Bai, J.-H. Yun, G. Liu, L. Wang, Advanced Materials, 30 (2018) 1800486.

[20] Y. Qiu, W. Liu, W. Chen, G. Zhou, P.-C. Hsu, R. Zhang, Z. Liang, S. Fan, Y. Zhang, Y. Cui, Science Advances, 2 (2016) e1501764.

[21] M. Dumortier, S. Haussener, Energy \& Environmental Science, 8 (2015) 3069-3082.

[22] S.A. Bonke, M. Wiechen, D.R. MacFarlane, L. Spiccia, Energy \& Environmental Science, 8 (2015) 2791-2796.

[23] J.T. Davis, D.V. Esposito, Journal of Physics D: Applied Physics, 50 (2017) 11.

[24] Y. Pihosh, I. Turkevych, K. Mawatari, J. Uemura, Y. Kazoe, S. Kosar, K. Makita, T. Sugaya, T. Matsui, D. Fujita, M. Tosa, M. Kondo, T. Kitamori, Scientific Reports, 5 (2015) 11141.

[25] S. Bell, G. Will, J. Bell, International Journal of Hydrogen Energy, 38 (2013) 6938-6947.

[26] A. Wolcott, W.A. Smith, T.R. Kuykendall, Y. Zhao, J.Z. Zhang, Small, 5 (2009) 104-111.

[27] A. Hankin, F.E. Bedoya-Lora, C.K. Ong, J.C. Alexander, F. Petter, G.H. Kelsall, Energy \& Environmental Science, 10 (2017) 346-360.

[28] F.E. Bedoya-Lora, A. Hankin, G.H. Kelsall, Journal of Materials Chemistry A, 5 (2017) 22683-22696.

[29] M.C.K. Sellers, E.G. Seebauer, Thin Solid Films, 519 (2011) 2103-2110. 
[30] J.H. Carey, B.G. Oliver, Nature, 259 (1976) 554-556.

[31] J. Qi, W. Zhang, R. Cao, Advanced Energy Materials, 8 (2018) 1701620.

[32] Y. Shi, Y. Yu, Y. Yu, Y. Huang, B. Zhao, B. Zhang, ACS Energy Letters, 3 (2018) 1648-1654.

[33] P. Yan, G. Liu, C. Ding, H. Han, J. Shi, Y. Gan, C. Li, ACS Applied Materials \& Interfaces, 7 (2015) 3791-3796.

[34] Y.J. Hwang, C. Hahn, B. Liu, P. Yang, ACS Nano, 6 (2012) 5060-5069.

[35] F.M. Pesci, G. Wang, D.R. Klug, Y. Li, A.J. Cowan, The Journal of Physical Chemistry C, 117 (2013) 25837-25844.

[36] A.P. Singh, A. Mettenbörger, P. Golus, S. Mathur, International Journal of Hydrogen Energy, 37 (2012) 13983-13988.

[37] Z. Fu, T. Jiang, Z. Liu, D. Wang, L. Wang, T. Xie, Electrochimica Acta, 129 (2014) 358-363.

[38] T. Li, J. He, B. Peña, C.P. Berlinguette, Angewandte Chemie International Edition, 55 (2016) 1769-1772.

[39] L. Hou, M. Zhang, Z. Guan, Q. Li, J. Yang, Applied Surface Science, 428 (2018) 640-647.

[40] O. Elbanna, M. Fujitsuka, S. Kim, T. Majima, The Journal of Physical Chemistry C, 122 (2018) 15163-15170.

[41] C. Fàbrega, S. Murcia-López, D. Monllor-Satoca, J.D. Prades, M.D. Hernández-Alonso, G. Penelas, J.R. Morante, T. Andreu, Applied Catalysis B: Environmental, 189 (2016) 133-140.

[42] H.S. Han, S. Shin, D.H. Kim, I.J. Park, J.S. Kim, P.-S. Huang, J.-K. Lee, I.S. Cho, X. Zheng, Energy \& Environmental Science, 11 (2018) 1299-1306.

[43] M. Radecka, M. Rekas, A. Trenczek-Zajac, K. Zakrzewska, Journal of Power Sources, 181 
(2008) 46-55.

[44] Q. Xu, J. Yu, J. Zhang, J. Zhang, G. Liu, Chemical Communications, 51 (2015) 7950-7953.

[45] J. Bandara, U.W. Pradeep, Thin Solid Films, 517 (2008) 952-956.

[46] Z. Chen, T.F. Jaramillo, T.G. Deutsch, A. Kleiman-Shwarsctein, A.J. Forman, N. Gaillard, R. Garland, K. Takanabe, C. Heske, M. Sunkara, E.W. McFarland, K. Domen, E.L. Miller, J.A. Turner, H.N. Dinh, Journal of Materials Research, 25 (2010) 3-16.

[47] K. Ding, B. Chen, Y. Li, Y. Zhang, Z. Chen, Journal of Materials Chemistry A, 2 (2014) 82948303.

[48] Y. Lin, G. Yuan, S. Sheehan, S. Zhou, D. Wang, Energy \& Environmental Science, 4 (2011) 4862-4869.

[49] A. Hameed, M.A. Gondal, Z.H. Yamani, Catalysis Communications, 5 (2004) 715-719. 
List of figures:

Figure 1. The diagram of energy level and configuration of PEC.

Figure 2. (a) The predicted current response under concentrated sunlight; (b) comparison between computational results and experimental data of photocurrent under various light intensities.

Figure 3. The relationship of photocurrent performance and $\mathrm{ABPE}$ at $0.4 \mathrm{~V}$ vs $\mathrm{Ag} / \mathrm{AgCl}$ with light intensity in circumstance of (a) limited by interfacial electrochemical kinetics; (b) mixed limiting by interfacial electrochemical kinetics and charge carrier transfer in semiconductor; (c) limited by charge carrier transfer in semiconductor; (d) summary of working regions for PEC.

Figure 4. (a) Light decay in the semiconductor; (b) profile of photocurrent performance and ABPE along with the thickness of semiconductor at $0.15 \mathrm{~V}$ vs $\mathrm{Ag} / \mathrm{AgCl}$; (c) the relationship between absorption fraction and wavelength.

Figure 5. (a) Photocurrent profiles at $0.4 \mathrm{~V}$ vs $\mathrm{Ag} / \mathrm{AgCl}$ against light absorption coefficient at various charge diffusion lengths; (b) maximum $\mathrm{ABPE}$ profiles at $0.15 \mathrm{~V}$ vs $\mathrm{Ag} / \mathrm{AgCl}$; (c) light absorption fraction plots with wavelength at various light absorption factor.

Figure 6. ABPE distribution of PECs consists of various photoanodes.

Figure 7. Theoretical ABPE map against band gap and condution band position. Band position (i) presents the VB at OER equilibrium potential and $\mathrm{CB}$ at HER equilibrium potential; band position (ii) presents the $\mathrm{CB}$ at $\mathrm{OER}$ equilibrium potential; band position (iii) presents the $\mathrm{CB}$ locates between the equilibrium potential of OER and HER. 
List of tables:

Table 1. Optical and charge transfer properties of $\mathrm{TiO}_{2}$ and $\mathrm{H}-\mathrm{TiO}_{2}$. 
Figure 1 The diagram of energy level and configuration of PEC.
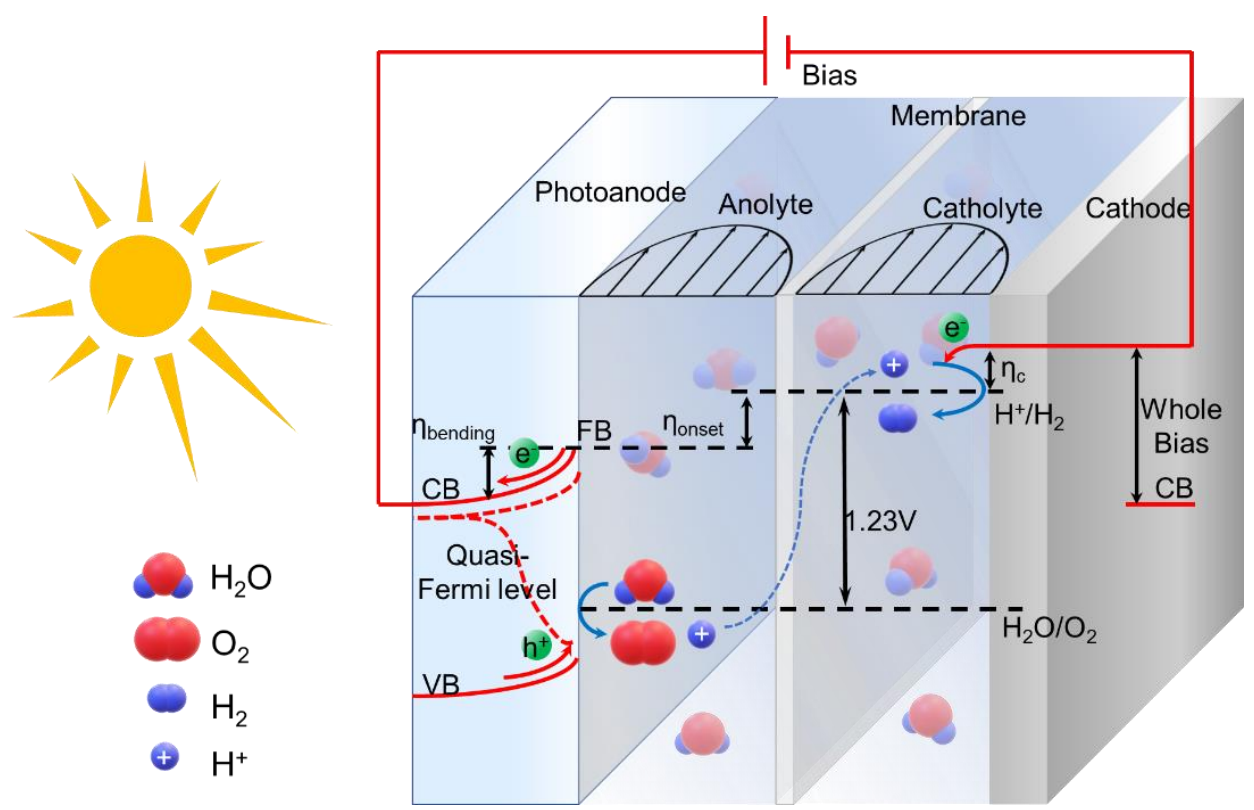
Figure 2 (a) The predicted current response under concentrated sunlight; (b) comparison between computational results and experimental data of photocurrent under various light intensities.
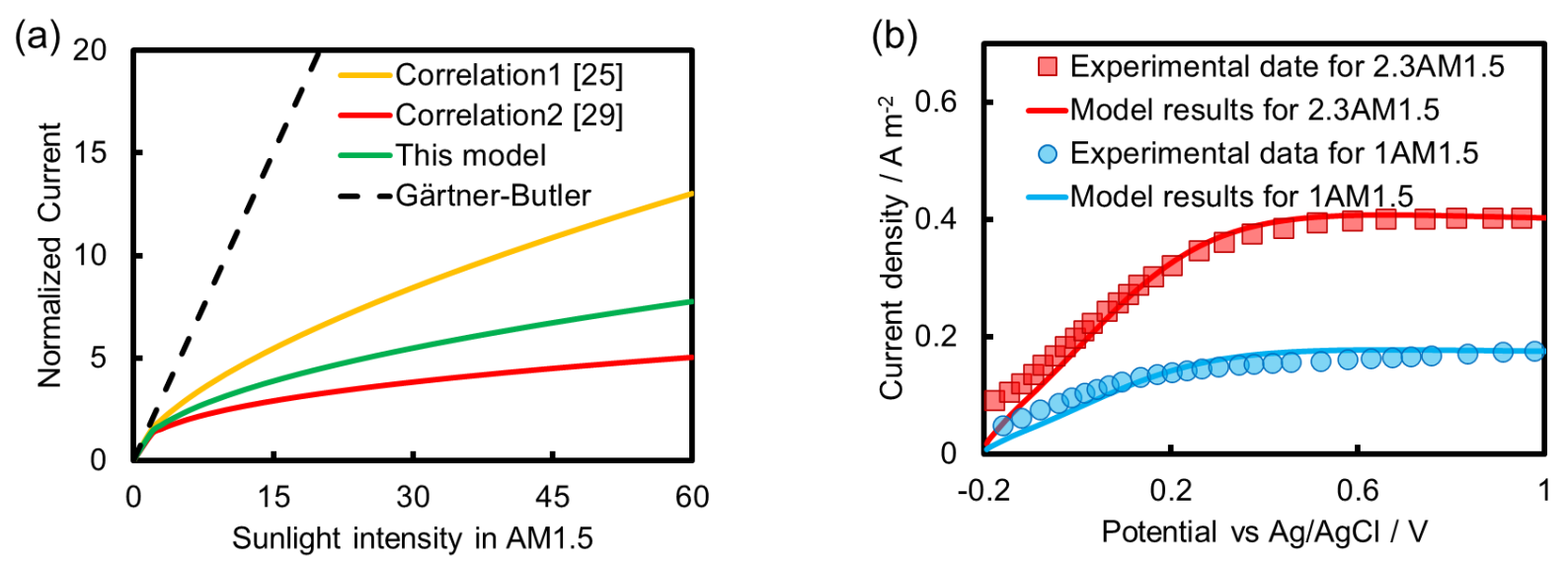
Figure 3. The relationship of photocurrent performance and $\mathrm{ABPE}$ at $0.4 \mathrm{~V} v \mathrm{Ag} / \mathrm{AgCl}$ with light intensity in circumstance of (a) limited by interfacial electrochemical kinetics; (b) mixed limiting by interfacial electrochemical kinetics and charge carrier transfer in semiconductor; (c) limited by charge carrier transfer in semiconductor; (d) summary of working regions for PEC.
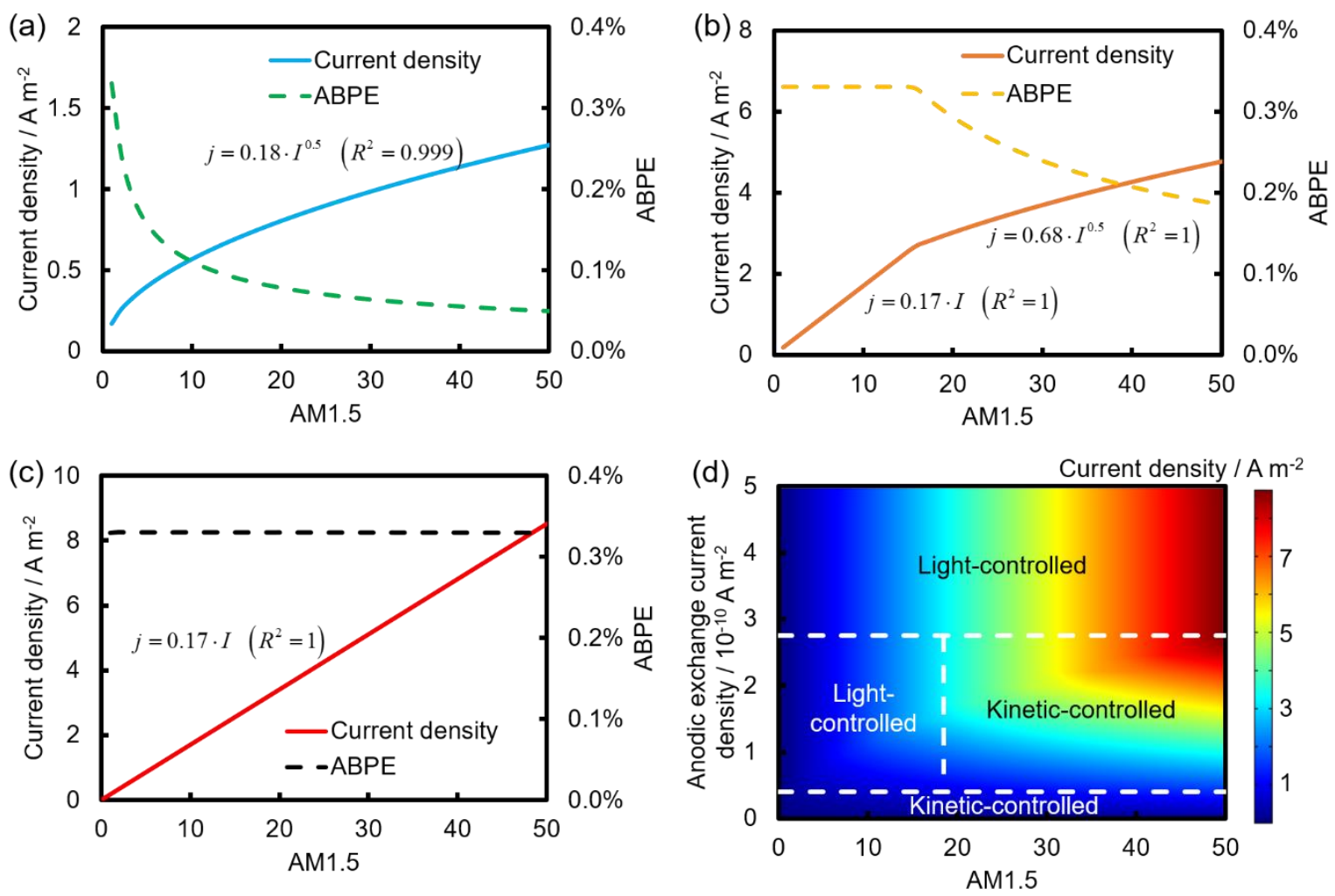
Figure 4. (a) Light decay in the semiconductor; (b) profile of photocurrent performance and ABPE along with the thickness of semiconductor at $0.15 \mathrm{~V}$ vs $\mathrm{Ag} / \mathrm{AgCl}$; (c) the relationship between absorption fraction and wavelength.

(a)
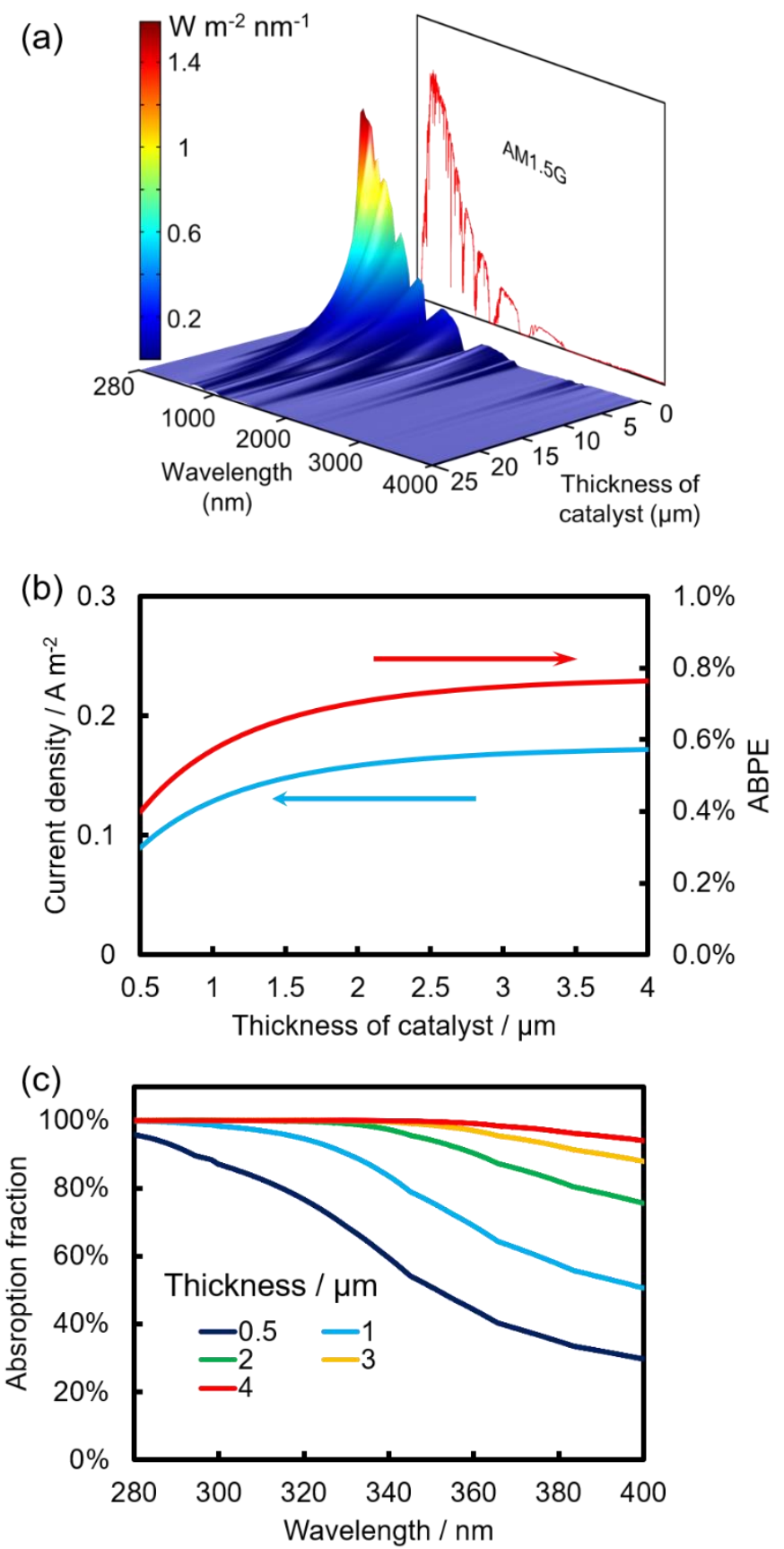
Figure 5. (a) Photocurrent profiles at $0.4 \mathrm{~V}$ vs $\mathrm{Ag} / \mathrm{AgCl}$ against light absorption coefficient at various charge diffusion lengths; (b) maximum $\mathrm{ABPE}$ profiles at $0.15 \mathrm{~V}$ vs $\mathrm{Ag} / \mathrm{AgCl}$; (c) light absorption fraction plots with wavelength at various light absorption factor.
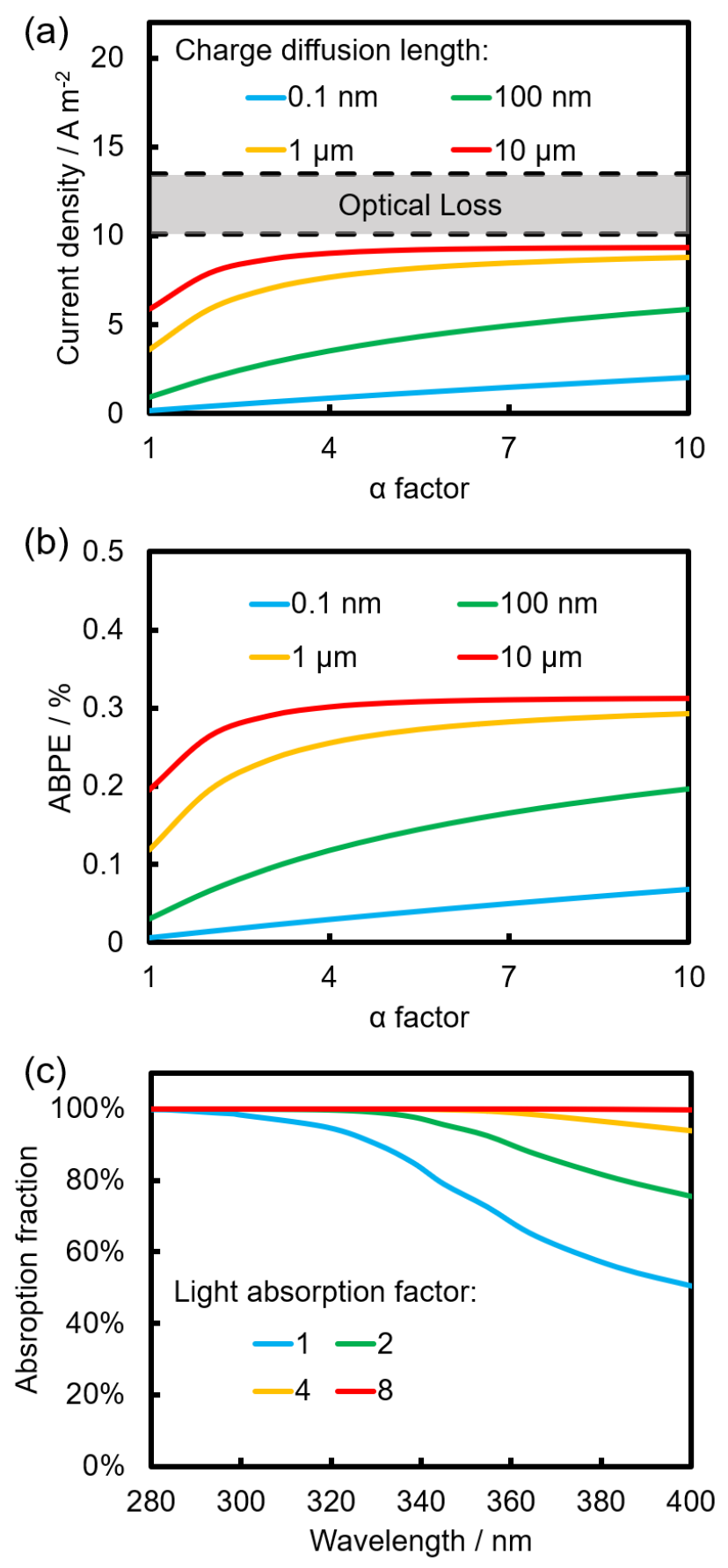
Figure 6. ABPE distribution of PECs consists of various photoanodes.

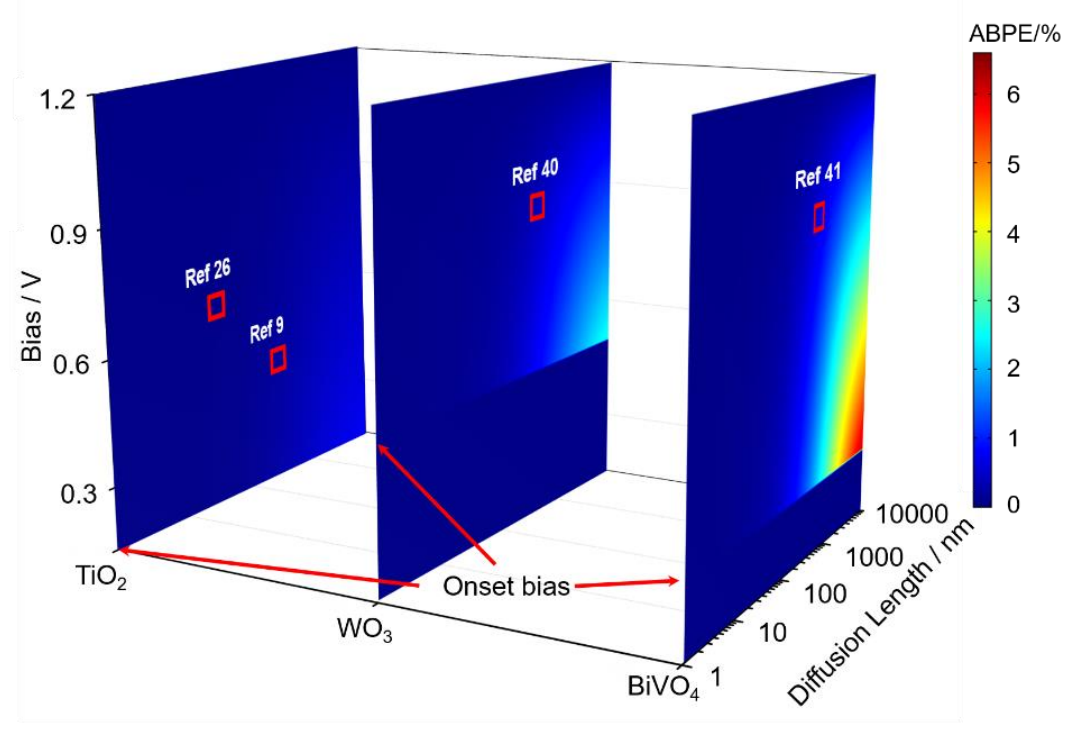


Figure 7. Theoretical ABPE map against band gap and condution band position. Band position (i) presents the VB at OER equilibrium potential and $\mathrm{CB}$ at HER equilibrium potential; band position (ii) presents the $\mathrm{CB}$ at $\mathrm{OER}$ equilibrium potential; band position (iii) presents the $\mathrm{CB}$ locates between the equilibrium potential of OER and HER.

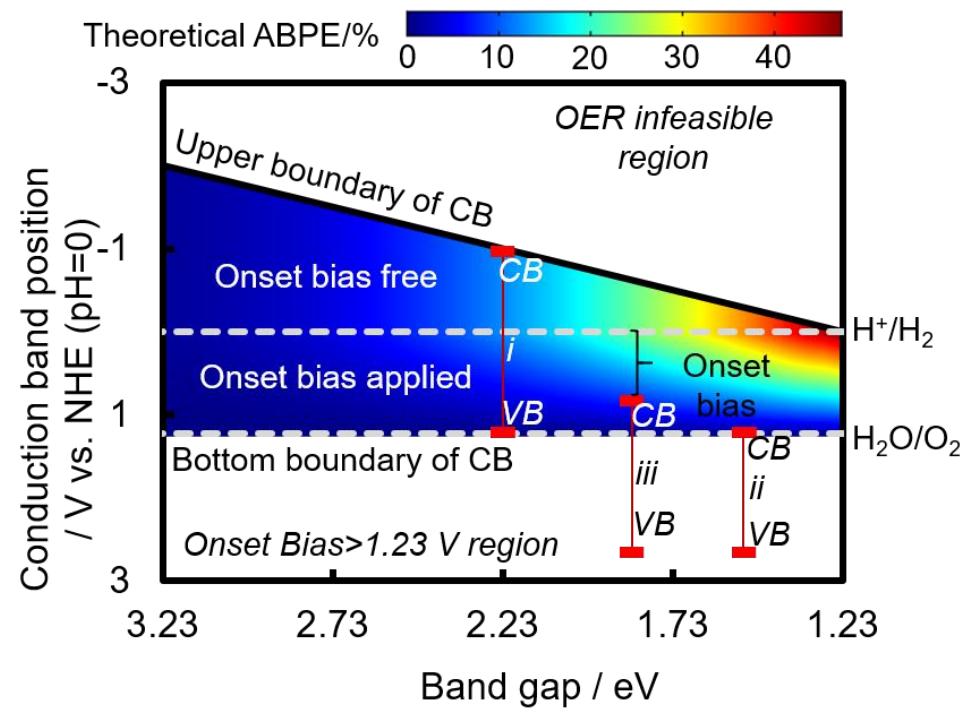

